\title{
Diseño de indicadores de gestión del networking para el emprendimiento universitario
}

\author{
Miguel Arroyo Rizo Patrón \\ Universidad de Lima. Lima, Perú \\ Correo electrónico: Marroyo@ulima.edu.pe
}

Recibido: 9 de febrero de 2014 / Aprobado: 1 de abril de 2014

Resumen: En el Perú existe un importante interés académico por el emprendedor. Esto se refleja en el desarrollo de programas de apoyo al emprendedor en el claustro universitario, cuyas metodologías de implementación involucran programas de incubación de ideas de negocios e insertarlos en el mercado por redes de apoyo (networking). Rara vez estos programas son sometidos a evaluaciones por medio de indicadores de gestión. Esta investigación buscó analizar los procedimientos de incubación universitaria en el medio, sus redes de apoyo y la inserción de sus startups en el mercado para aplicarlo en un diseño de indicadores de gestión para los startups en la Universidad de Lima.

Palabras clave: emprendedores / redes de apoyo / indicadores de gestión / ecosistema emprendedor

\section{Design of management indicators networking for university entrepreneurship}

\begin{abstract}
Aвstract: In Peru there is a significant academic interest in entrepreneurship. This is reflected in the development of programs to support entrepreneurship at the university cloister. The implementation methodology in national programs involving universities incubation of business ideas of their students to make them startups and insert them into the market. A feature of such programs is that they rarely undergo evaluations to provide feedback through management indicators. This research sought to analyze university procedures incubation in the medium. To then apply it in a design management indicators for startups at the University of Lima.
\end{abstract}

Key words: entrepreneurs / network support / management indicators / entrepreneurial ecosystem 


\section{INTRODUCCIÓN}

Con respecto a la formación emprendedora en las universidades, según Freyre (2004), se pueden considerar tres niveles o capas mentales que debemos inculcar en las personas a quienes deseamos convertir en emprendedores:

El primer nivel o más externo corresponde a los hábitos técnicos, que son fácilmente modificables. En este nivel entraría todo aquello que por medio de la educación puede lograrse para que una persona aprenda a ser emprendedor.

Por ejemplo, las herramientas que le permitan elaborar un plan de negocios.

En la otra punta está lo más profundo del emprendedor, la capa neuronal de los talentos, inmodificables, que se pueden tener o no. No hay manera de que se pueda trabajar sobre ello.

En este extremo se considera que si una persona no tiene talentos propios para ser emprendedor, podrá aprender superficialmente alguna herramienta, pero nunca será un emprendedor nato, que lograra ver los problemas y oportunidades de la vida real para transformarlos en negocios.

En el medio está la capa de las metahabilidades. Estas se modificarán y se adquirirán si el emprendedor realmente lo desea. Por ejemplo: dirigir su propio aprendizaje continuo, desarrollar autoestima y su toma de decisiones, asumir el riesgo para lograr su independencia, aprender a vivir y tomar de la mejor manera posible y apasionarse por su proyecto.

\section{LAS INCUBADORAS UNIVERSITARIAS EN EL PERÚ}

La incubadora universitaria convierte la idea de negocio en un startup, en el cual debe cortar figuradamente su cordón umbilical con el incubado para que se inserte en el mercado.

Las redes de apoyo, creadas por la incubadora, dependen de la cultura, la misión, los objetivos, la historia y la tradición de la universidad 
huésped, y pasar luego por las siguientes etapas al insertarse en el mercado:

- Umbral de credibilidad (Aceituno \& Cáceres, 2009). En esta etapa el startup debe conseguir su capital semilla, el armado de su organización en cuanto al personal y la maquinaria productiva. Una vez con ellos puede conseguir los otros recursos para completar su capacidad productiva. Esta situación depende mucho de las redes de apoyo existentes para el emprendedor, como se ve en la figura 1, pues de no conseguir los recursos necesarios, el emprendedor se vería limitado en sus posibilidades de éxito al ingresar en el mercado.

- Umbral de sostenibilidad. Una vez que la empresa ha pasado exitosamente el umbral de credibilidad, puede afrontar una situación crítica determinada por el nivel de ingresos y beneficios que es capaz de obtener en su actividad comercial. La superación del umbral de sostenibilidad se consigue en función de la capacidad empresarial de los emprendedores para reconfigurarse continuamente con sus recursos y capacidades disponibles ante los retos del entorno.

Figura 1

Redes de apoyo en una incubadora de negocios

Factores que influyen en la generación de spin off académicas

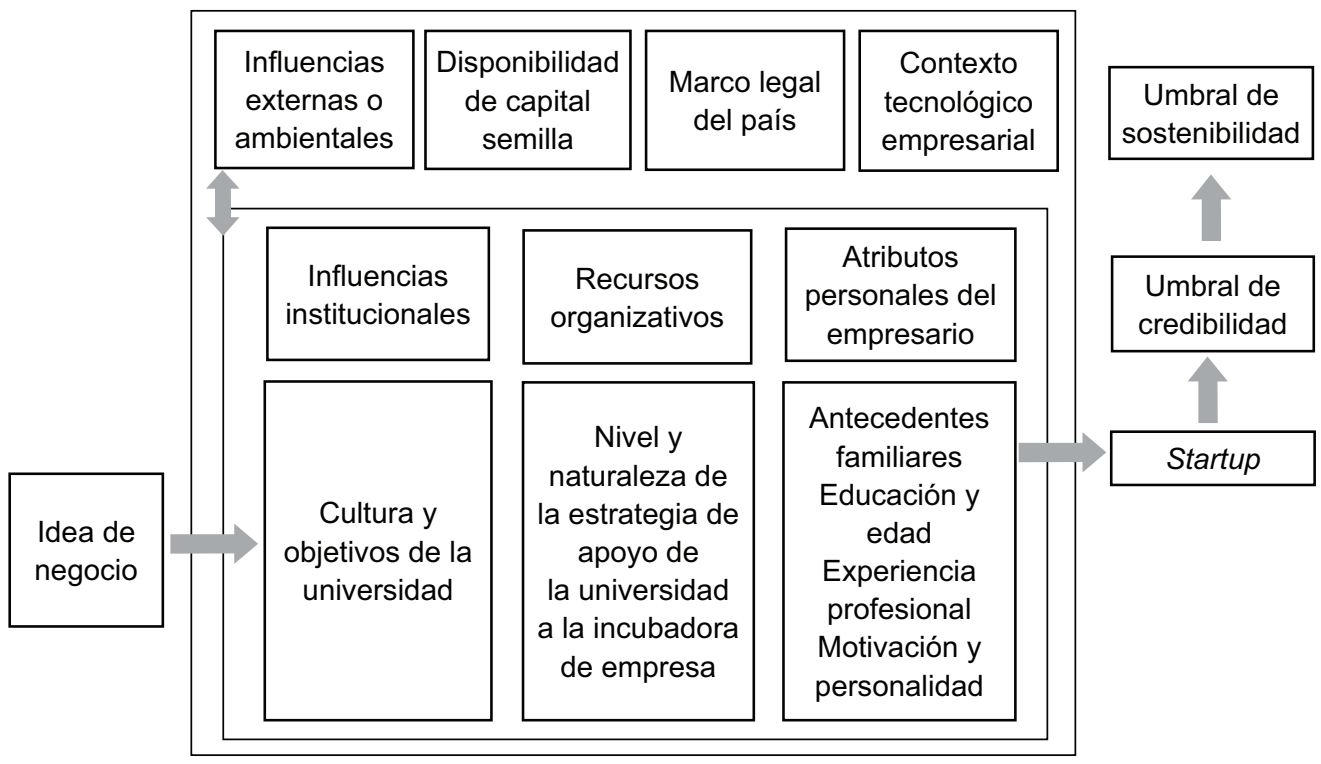

Elaboración propia. 


\section{PROCEDIMIENTO DE ESTA INVESTIGACIÓN}

La investigación tuvo como hipótesis principal que el éxito de los emprendimientos incubados en las instituciones universitarias se basa en una adecuada formación de sus redes de apoyo. Para determinar el sistema de trabajo del networking de dichas incubadoras se buscó una asociación que las acogiera, que fue la asociación peruana de incubadoras de empresas Perú Incuba, que reúne a las principales incubadoras universitarias del país.

Como resultado de esta investigación se encontró que los sistemas de incubadoras universitarias peruanas tienen una conformación típica, como se muestra en la figura 2, donde se puede apreciar que poseen como primera unidad un Banco de Ideas de Negocios (los negocios son promovidos por medio de concursos de la propia universidad o externos a esta).

La segunda unidad es un Centro de Preincubación, cuyo ingreso en algunos casos es libre y en otros es selectivo. En la mayoría de las unidades de preincubación universitarias no se cobra a sus usuarios. Se espera que en esta unidad concreten sus planes de negocios, para luego pasar al proceso de incubación.

Como tercera unidad se encuentra la Incubación, a la cual se accede con los planes de negocios para su puesta en marcha. Allí reciben asesoría especializada por medio de profesores asesores y son dirigidos por un profesor tutor, que virtualmente se convierte en un gerente de proyecto. En esta etapa, la mayoría de las universidades cobra a los emprendedores. Una vez que la empresa se gradúa, esta se encuentra formalizada y en su mercado.

Actualmente no existen en el sistema mecanismos de apoyo para un proceso de spin off posterior en el umbral de sostenibilidad. 

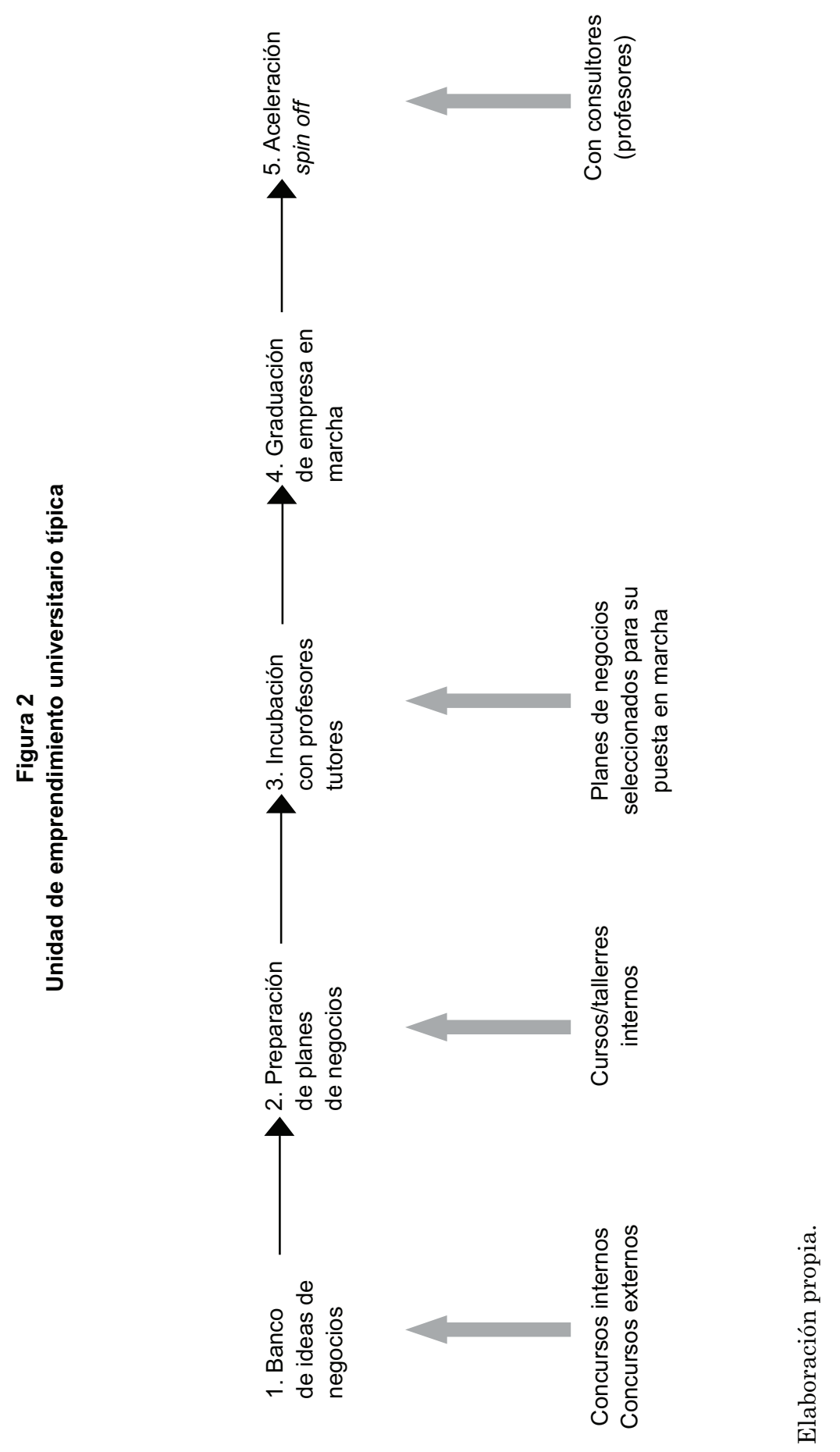


\section{BASES TEÓRICAS PARA EL DISEÑO DE INDICADORES DE GESTIÓN}

Con el fin de obtener los indicadores de gestión de un networking exitoso para los emprendimientos universitarios apoyados por el sistema de incubación, se realizó un estudio de sistemas similares en las instituciones de educación del país que cuentan con este tipo de incubadoras.

Se encontraron los aspectos esenciales de su conformación sobre la base de un benchmarking y con la ayuda de una herramienta conocida como balanced scorecard, para plantear los indicadores necesarios para sus redes de apoyo. Asimismo, se controló el proceso, dividiendo los indicadores en cuatro dimensiones: aprendizaje y crecimiento, inserción en mercado, obtención de capital semilla y formalización; de esta manera se obtuvo una calificación general de la calidad de redes de apoyo.

En general, para la evaluación del desempeño de la gestión como formadores de emprendedores se debe contar con indicadores, con los que podemos efectuar el seguimiento y la evaluación periódica de nuestra organización en su estructura formativa, se entiende que los indicadores encontrados tienen, al menos, tres funciones básicas (Sedecap, 1997):

- Descriptiva, que consiste en la aportación de información sobre el estado real de nuestro programa de formación de emprendedores.

- Valorativa, que consiste en añadir, a esa información, un juicio de valor lo más objetivo posible sobre si el resultado obtenido es adecuado o no.

- Causal, que consiste en identificar las causas del menor, mayor o igual resultado esperado respecto a las metas propuestas en el logro del startup de nuestros emprendedores.

Según los estudios tendientes a la conformación de indicadores de gestión en Estados Unidos y Europa (Sedecap, 1997), las principales asociaciones de incubadoras existentes en el mundo son la National Business Incubation Association (NBIA), en Estados Unidos, y la European Business Innovation Centres Network (EBN), en Europa. 
Las incubadoras que presentan mayores indicadores de éxito según The Centre for Strategy \& Evaluation Services LLP UK (CSES), en su estudio "Benchmarking of Business Incubators, Final report", son aquellas que cumplen las siguientes condiciones:

- Están diseñadas para apoyar y ser parte de una estrategia más amplia orientada a una determinada política.

- Son entidades que trabajan de manera conjunta con otras organizaciones para promover estrategias comunes.

- Son promovidas por una asociación de agentes del sector público y privado, constituido por un amplio rango de organizaciones que provienen tanto de sectores privados como públicos, incluidas las autoridades locales, universitarias, compañías e instituciones financieras. Las autoridades públicas desempeñan una importante función catalítica y de dirección, proporcionando una inversión crucial durante la fase de desarrollo de la incubadora.

La NBIA es la principal organización mundial de incubación de negocios emprendedores. Su misión es contribuir en la formación y ser centro de intercambio de datos en la dirección y desarrollo de incubadoras, así como servir de herramienta para la asistencia de startups y empresas de reciente creación.

Esta asociación considera que hay tres aspectos fundamentales que se deben tener en cuenta cuando se desarrolla un sistema de recolección de datos para indicadores de gestión:

- Incluir sobre todo empresas graduadas.

- Es fundamental entender la importancia de crear una base de datos.

- Los datos recopilados deben ayudar a la industria a demostrar su valor.

Es necesaria una recopilación anual para los incubados y las empresas graduadas en un horizonte de cinco años después de que hayan dejado el programa, información suficiente para demostrar su permanencia en el mercado.

Por su parte, la asociación EBN, fundada en 1984, tiene su base en Bruselas. Ofrece un servicio de coordinación de actividades de los centros de incubación, desarrollando y promoviendo este concepto en todos los países de la Unión Europea. 
Los indicadores claves, también llamados indicadores de éxito, representan una parte fundamental de las herramientas de control de las incubadoras asociadas para definir sus objetivos medibles.

Estos indicadores, que han sido seleccionados por el método Specific, measurable, achievable, result-oriented, time-bound (SMART), ayudan a las incubadoras a evaluar los objetivos que se están consiguiendo y dónde se están implementando mejor sus estrategias. Son medidos durante cuatro años después del startup.

\section{EL CAPITAL SEMILLA EN NUESTRO ECOSISTEMA EMPRENDEDOR}

Los emprendedores formados en las incubadoras universitarias tienen un gran potencial para contribuir al crecimiento de la economía peruana, pero no podrán hacerlo si no cuentan con la financiación que les permita pasar de emprendedor a empresario; para ello, deben buscar maneras innovadoras de construir su negocio con acceso limitado al capital.

El Capital Semilla es un financiamiento inicial (fondos que no deben ser devueltos), para la creación de una microempresa o para permitir el despegue y/o consolidación de una actividad empresarial existente. Una vez que el proyecto ya está instalado y funcionando, se puede recurrir a otras líneas de financiamiento para hacer crecer el negocio, como por ejemplo, a través del Capital de Riesgo (Banca Fácil, 2012).

Algunas universidades cuentan con fondos propios para financiar los proyectos de negocios de sus estudiantes. Por ejemplo, la Universidad de Lima lleva a cabo el concurso "El primer paso", donde los alumnos presentan ideas de negocios; se escogen las 30 mejores, las que ingresan a un proceso de aceleración empresarial, con un asesoramiento personalizado para el desarrollo de sus planes de negocios por un periodo de 45 días; luego, se seleccionan las 10 mejores propuestas.

También existen los fondos subsidiados, que se otorgan por medio de concursos nacionales e internacionales, como los de Fundación Romero ("Para quitarse el sombrero"), Wayra Perú, Nescafé, entre otros. 
Otro sistema para conseguir capital semilla es el desarrollado por programas internacionales como el Road Show Startup, el Startup Weekend y el Founder Institute, que permite vincularse con los inversionistas privados llamados Business Angeles.

La inversión por parte de los Business Angeles ofrece una solución alternativa a las altas tasas de interés que enfrentan los créditos bancarios y las garantías que se deben presentar para obtenerlos; además, crean una sinergia del sistema, como se muestra en la figura 3.

\section{Figura 3}

Sistema financiero sinérgico de los Business Angeles

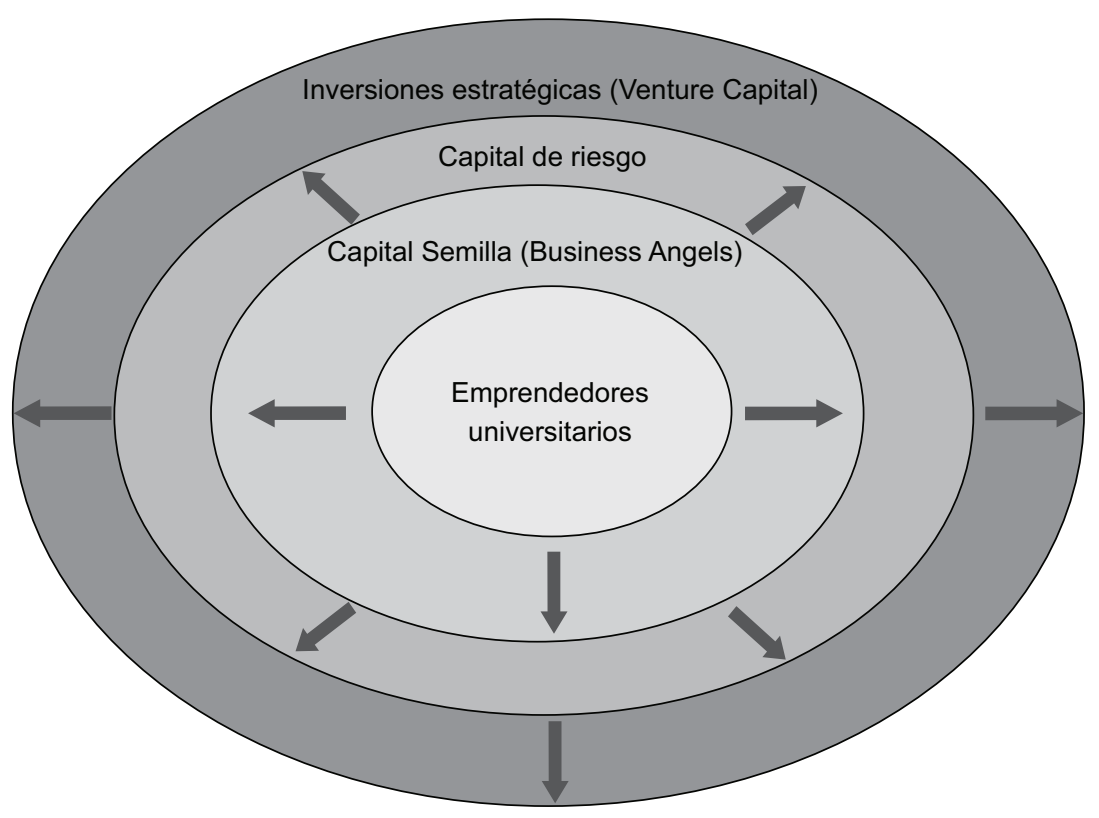

El mapa estratégico en la incubación universitaria (Kaplan \& Norton, 2004). 


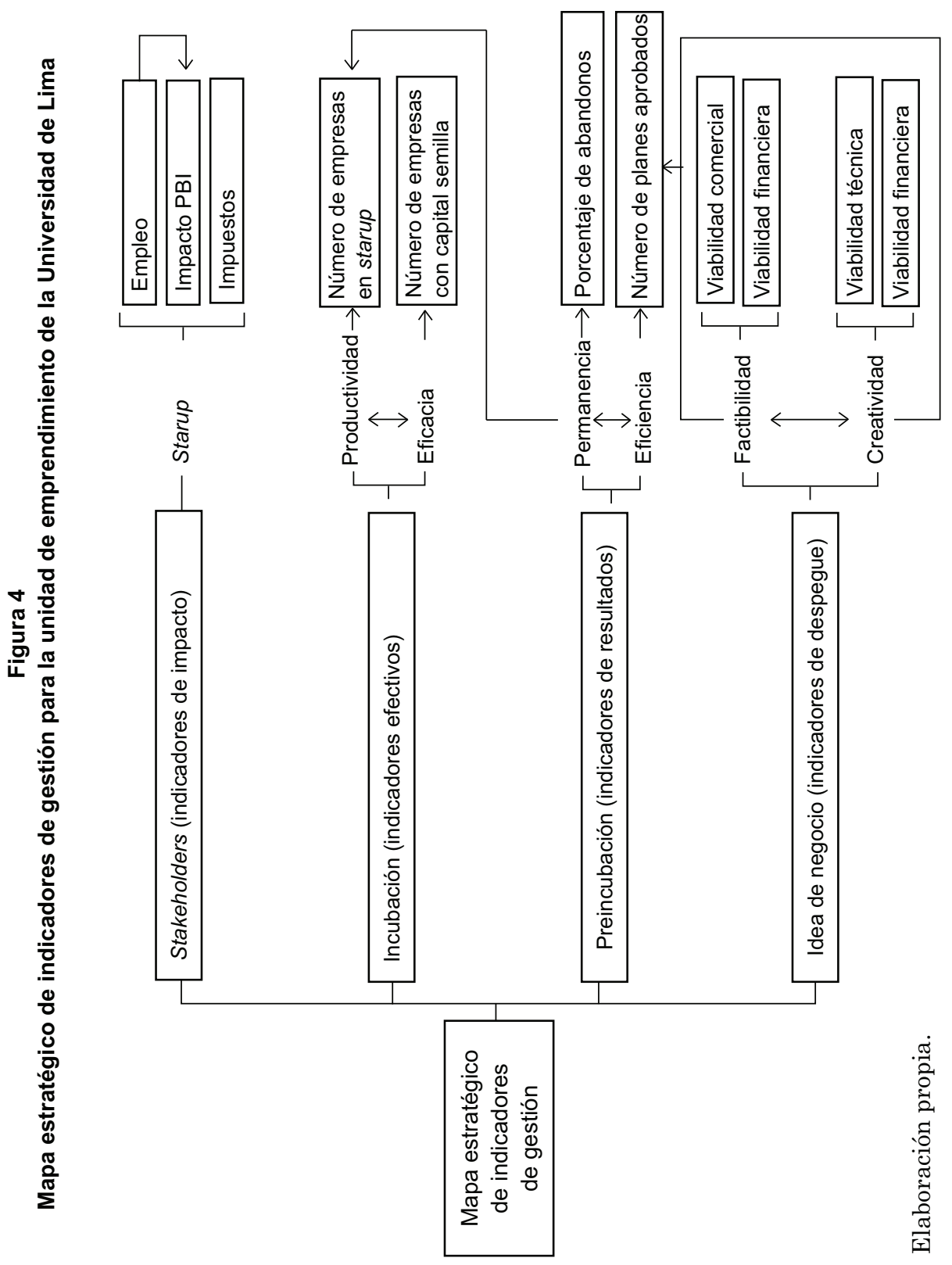


En el primer nivel de la idea de negocio están los indicadores de despegue: factibilidad (cuantitativo) y creatividad (cualitativo).

Estos indicadores están relacionados con los subindicadores de viabilidad comercial, viabilidad financiera, viabilidad técnica y viabilidad administrativa.

A su vez, estos se encuentran interrelacionados con un segundo nivel para la preincubación: los indicadores de resultados: permanencia (cuantitativo) y eficiencia (cuantitativo).

En un tercer nivel figuran los indicadores de efecto para la incubación: productividad (número de empresas logradas en startups) y eficiencia (número de empresas formadas con capital semilla).

Este tercer nivel genera en los stakeholders indicadores de impacto por interrelación: niveles de empleo logrado en las empresas incubadas, impacto en la generación del PBI, nivel de pago de impuestos, entre otros.

\section{APLICACIÓN DE LOS INDICADORES DE GESTIÓN ENCONTRADOS}

La gestión de una incubadora de negocios universitaria debe medirse por el tamaño del embudo, es decir, los ratios de caída desde las ideas de negocios hasta los startups logrados.

Es importante la conformación, por parte de la incubadora, de una base de datos actualizados de las empresas en proceso de formación y de las ya graduadas. En el caso de la Universidad de Lima, su formato de apoyo al emprendimiento está basado en un sistema de recojo de datos del avance del proceso por una plataforma virtual, que ayuda al proceso de información; pero aún no se ha trabajado el sistema para recabar información de las empresas graduadas. 
Gráfico 5

Esquema del sistema actual de emprendimiento en la Universidad de Lima

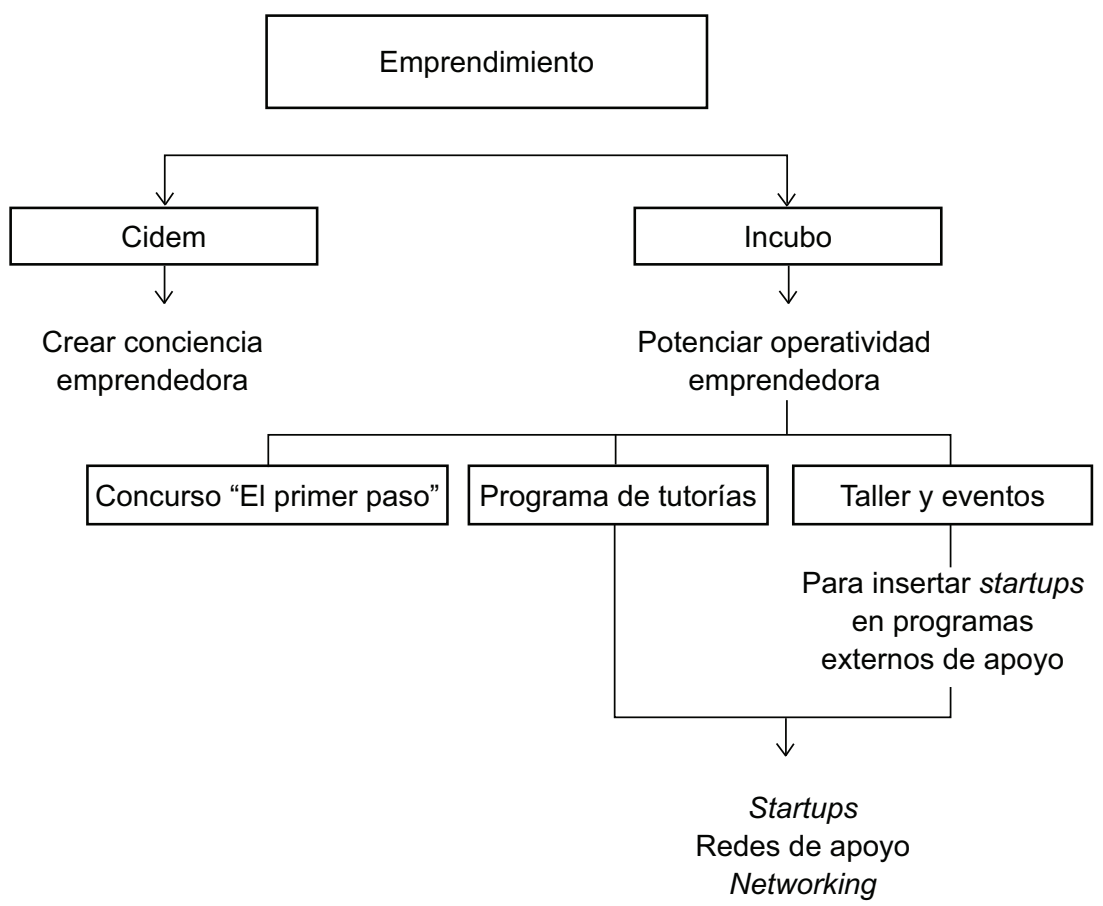

Elaboración propia.

\section{PRESENTACIÓN DE LOS RESULTADOS DE LA INVESTIGACIÓN}

Objetivos principales y secundarios. El objetivo principal fue comparar los indicadores encontrados con la experiencia en el sector de los asistentes y evaluar su aplicabilidad, y el secundario el de recibir sus aportes para mejorar en la investigación la presentación y aplicabilidad de los indicadores en su fondo y en su forma.

Público asistente. Para un conversatorio sobre el tema se invitó a este taller a ejecutivos y profesores tutores de la Oficina de Emprendimiento de la Universidad de Lima, profesores principales de la Facultad de Economía de la Escuela de Negocios de esta misma universidad, y a ejecutivos de centros de incubación de otras universidades. 
Procedimiento del conversatorio. En el conversatorio se presentó una propuesta de indicadores de gestión en función de la arquitectura del networking, y en un mediano plazo para los startup, logrados por la Oficina de Emprendimiento de la Universidad de Lima, para ser discutida por los asistentes, con cuyos aportes se llegó a los indicadores propuestos en las tablas 1 y 2 .

Tabla 1

Indicadores propuestos para el sistema al I nivel startup

\begin{tabular}{llcl}
\hline \multicolumn{1}{c}{ Nivel } & Indicador & Tipo & \multicolumn{1}{c}{ Medición } \\
\hline $\begin{array}{l}\text { Concurso } \\
\text { "El primer paso" }\end{array}$ & Factibilidad & Cualitativo & $\begin{array}{l}\text { Ideas aprobadas / ideas } \\
\text { presentadas }\end{array}$ \\
Programa tutorías & Eficiencia & Cualitativo & $\begin{array}{l}\text { Startups / No de emprendedores } \\
\text { al inicio del programa }\end{array}$ \\
$\begin{array}{l}\text { Talleres y eventos } \\
\text { de inserción }\end{array}$ & Eficiencia & Cualitativo & $\begin{array}{l}\text { No de empresas que logran } \\
\text { capital semilla / No de empresas } \\
\text { asistentes }\end{array}$ \\
\hline
\end{tabular}

Elaboración propia.

Tabla 2

Indicadores para la etapa de inserción

\begin{tabular}{lccc}
\hline \multicolumn{1}{c}{ Nivel } & Indicador & Tipo & Medición \\
\hline $\begin{array}{l}\text { Empresas graduadas a 4 } \\
\text { años de inserción }\end{array}$ & Éxito comercial & Cualitativo & $\begin{array}{l}\text { Facturación anual / } \\
\text { Sobre capital social }\end{array}$ \\
$\begin{array}{l}\text { Empresas graduadas a 4 } \\
\text { años de inserción }\end{array}$ & Sombra Social & Cualitativo & $\begin{array}{l}\text { Empleos creados / } \\
\text { Sobre capital invertido }\end{array}$ \\
\hline
\end{tabular}

Elaboración propia. 


\section{CONCLUSIONES}

Las incubadoras universitarias son parte integrante de las universidades que las sustentan; por lo tanto, al definir sus políticas, se subordinan a los objetivos corporativos, estratégicos y a la propuesta de valor que la universidad quiere implantar.

Las universidades son conscientes de que uno de los principales problemas para la inserción de sus startups en los mercados en forma exitosa es la necesidad de contar con redes de apoyo en el denominado umbral de credibilidad, cuando se busca el capital semilla.

Los concursos internos en las universidades sobre ideas de negocios no deben ser motivados solo por el premio, sino por el hecho de desarrollar la idea de negocio, hasta el punto de formar una empresa.

La cultura emprendedora universitaria debe poseer los elementos conformantes necesarios para generar el compromiso del emprendedor con su idea de negocio, de manera que incluso lo lleve a invertir fondos propios en su emprendimiento.

El tema del emprendimiento en una universidad debe contemplar tres vertientes: formación, investigación y extensión; ello requiere generar indicadores de gestión para medir cuantitativa y cualitativamente los avances en estas tres vertientes a través del tiempo.

La gestión de una incubadora de negocios universitaria debe medirse por el tamaño del embudo, es decir, los ratios de caída desde las ideas de negocios hasta los startups logrados, por medio de indicadores de gestión, como los propuestos en esta investigación.

Es importante contar, por parte de la incubadora, con datos actualizados de las empresas ya graduadas. En función a este sistema se puede obtener información periódica de dichas empresas para nuestros indicadores de gestión.

En el caso de las empresas graduadas, se aconseja un periodo de por lo menos cuatro años después del startup para medir su supervivencia y desempeño, es decir, su comportamiento en el umbral de sostenibilidad. 


\section{REFERENCIAS}

Aceituno, T., \& Cáceres, R. (2009). Elementos para elaboración de un marco de análisis para el fenómeno de las spin-offs universitarias. Revista de Economía Mundial, 23, 23- 51.

Althaus, J. de. (2007). La revolución capitalista en el Perú. Lima: Fondo de Cultura Económica.

Ballón, E. (2008). La cohesión social desde América Latina y el Caribe. Recuperado de http://www.eclac.cl

Banca Fácil (noviembre de 2012). ¿Qué es el Capital Semilla? Banca Facil (página web). Recuperado de http://bancafacil.cl/bancafacil/ servlet/contenido?indice $=1.2 \&$ dpublicacion

Béjar, F. (2004). Propuesta de indicadores para la medición del impacto económico de la Red Viveros de la Ciudad de Madrid. Madrid: Trikarty.

Beraza, J., \& Rodríguez, A. (2011). Los programas de apoyo a la creación de spin-offs en las universidades españolas: una comparación internacional. Investigaciones Europeas de Dirección y Economía de la Empresa, 17, 89-117.

Brown, B., Butler, J., \& Chamornmarn, W. (s. f.). Informational networks, entrepreneurial action and performance. Asia Pacific Journal of Management, 20, 151-174.

Castro, S. (2008). A la velocidad del byte. Medellín: Escuela de Administración, Finanzas y Tecnología, Fondo Editorial.

Consejo Nacional de Ciencia, Tecnología e Invocación Tecnológica. (2011). Encuentro Científico Internacional. Lima: Autor.

Freyre, A. (2004). Pasión por emprender: de la idea a la cruda realidad. Barcelona: Granica.

Kaplan, R., \& Norton, D. (febrero, 2004). Strategy maps: converting intangible assets into tangible outcomes. Harvard Business Review Press.

Osterwalden, A., \& Pigneur, Y. (2011). Generación de modelos de negocios. Barcelona: Ediciones Deusto. 
Secretaria de Desarrollo de Evaluación y Control (Sedecap). (1997). Guía técnica para el diseño y construcción de indicadores para evaluar la acción del Gobierno. Puebla: Gobierno de Puebla.

Steiner, G. (2004). Planeación estratégica: lo que todo director debe saber. México: Compañía Editorial Continental.

Taylor, S. J. (1990). Introducción a los métodos cualitativos de investigación: La búsqueda de significados. Buenos Aires: Paidós 